\title{
Targeted Editing of SIMAPK6 Using CRISPR/Cas9 Technology to Promote the Development of Axillary Buds in Tomato Plants
}

\author{
Yunzhou Li $\mathrm{Li}^{1, \dagger}$, Ningbo Yue ${ }^{1, \dagger}$, Abdul Basit ${ }^{1}$, Yulong $\mathrm{Li}^{2}$, Dalong Zhang ${ }^{3}$, Lei Qin ${ }^{4}$, M. J. C. Crabbe ${ }^{5,6,7}$, Wen Xu ${ }^{1}$, \\ Yong Wang ${ }^{1} \&$ Jianmin Yan $^{1}$ \\ ${ }^{1}$ College of Agriculture, Guizhou University, Guiyang, Guizhou, China \\ ${ }^{2}$ College of Natural Resources and Environment, Northwest A\&F University, Yangling, Shaanxi, China \\ ${ }^{3}$ College of Horticultural Science and Engineering, Shandong Agricultural University, Tai'an, Shandong, China \\ ${ }^{4}$ College of Horticulture, Northeast Agricultural University, Harbin, Heilongjiang, China \\ ${ }^{5}$ Wolfson College, Oxford University, Oxford, UK \\ ${ }^{6}$ Institute of Biomedical and Environmental Science and Technology, University of Bedfordshire, Luton, UK \\ ${ }^{7}$ School of Life Science, Shanxi University, Taiyuan, China \\ Correspondence: Yunzhou Li, College of Agriculture, Guizhou University, Guiyang 550025, China. Tel: \\ 86-158-9139-7294. E-mail: liyunzhou2007@126.com
}

Received: November 8, 2020

Accepted: December 15, $2020 \quad$ Online Published: January 15, 2021

doi:10.5539/jas.v13n2p11

URL: https://doi.org/10.5539/jas.v13n2p11

${ }^{\dagger}$ These authors contributed equally to this work.

The research is financed by National Natural Science Foundation of China (No. 32060679; No.31760594; No. 31960604), Research Project on Talent Introduction of Guizhou University (No. [2017]50) and National Natural Fund Cultivation Project of Guizhou University (No. [2017] 5788-28).

\begin{abstract}
The mitogen-activated protein kinase (MAPK) cascade signaling system has been relatively conserved throughout the evolution of eukaryotes and is involved in the regulation of growth and development and metabolism. In this study, dwarf tomato plants were used as the research material. First, the tissue-specific expression of SIMAPK6 was measured in wild-type plants by quantitative RT-PCR. The results showed that SIMAPK6 was highly expressed in the tissues of the stems, leaves and flowers but was expressed at low levels in the tissues of the roots, sepals and fruits. Second, SIMAPK6-knockout lines CRISPR-3 and CRISPR-7 were obtained by CRISPR-Cas9 technology and Agrobacterium-mediated transformation. Compared with wild-type, the mutant lines CRISPR-3 and CRISPR-7 showed significant phenotypic characteristics, such as increased numbers of axillary buds and true leaves, thickened stems, and longer leaflets. In addition, to explore the molecular mechanism by which MAPK regulates axillary bud growth, we also showed that SIMAPK6 positively regulates the strigolactone synthesis genes SlCCD7 and SlCCD8 and the gibberellin (GA) synthesis genes GA20ox3 and GA3oxl and negatively regulates the axillary bud development-related genes $L s, B L$ and $B R C 1 b / T C P 8$ and the GA synthesis inhibitory gene GAI. Therefore, SIMAPK6 appears to regulate the synthesis of strigolactone and GA to induce the growth and development of tomato axillary buds.
\end{abstract}

Keywords: axillary buds, gene knockout, SIMAPK6, tomato

\section{Introduction}

Plant morphogenesis has great influence on yield and quality, the number of side branches is very important index to plant architecture. The lateral branches develop from the axillary buds. Many factors, especially hormones, determine the development of axillary buds (Huh et al., 2013; Chen et al., 2016). Strigolactones and gibberellin (GA), two kinds of plant hormones, are reported to regulate shoot branching, stems elongation, plant growth and architecture (Yamaguchi et al., 2008; Vogel et al., 2010; Kohlen et al., 2012). In tomato, CAROTENOID CLEAVAGE DIOXYGENASE8 (SLCCD8) and CAROTENOID CLEAVAGE DIOXYGENASE7 ( $S C C D 7)$ are two key strigolactone biosynthesis-related genes. GA20oxs are essential 
enzymes for GA biosynthesis and control the GA content in different plant species. GA3oxs produce bioactive GAs, such as GA1, GA3, GA4 and GA7, in the final step (Yamaguchi et al., 2008).

The crosstalk between mitogen-activated protein kinases (MAPKs), and hormones, secondary messengers in plant signaling systems facilitates plant to adapt and survive in different environmental, including controlling plant architecture (Smekalova et al. 2014). MAPK cascade signaling system has been relatively conserved throughout the evolution of eukaryotes (Widmann et al., 1999; Group et al., 2002; Xu et al., 2015) and is involved in the regulation of the responses to various biotic and abiotic stresses, growth and development, and metabolism. MAPKs play an important role in the signaling pathway of eukaryotes. The MAPK signaling system is usually composed of tertiary protein kinases, including MAPK, MAPK kinase (MAPKK) and MAPKK kinase. MAPKs acts downstream of the tertiary protein kinase signaling system.

A total of 16 MAPKs have been identified in tomato, which can be further divided into four categories: A, B, C, and D (Kong et al., 2012). Among them, MAPK1, MAPK2 and MAPK3 belong to category A; these MAPKs have been the subject of many studies and are involved in the regulation of various stresses in plants (Stulemeijer et al., 2007; Zhou et al., 2014; Lv et al., 2017). The remaining 13 MAPKs belong to categories B, C, and D, which have been poorly studied. Among them, tomato MAPK6 (SIMAPK6) belongs to category B and has a loop containing a MEY motif. However, the function of SIMAPK6 is still unknown.

Genome-editing technology has been used in Arabidopsis, tomato, rice, apple, watermelon, potato, soybean, maize, wheat, sorghum and grape (Feng et al., 2014; Ito et al., 2015; Wang et al., 2016; Reem et al., 2019; Char et al., 2019a; Nishitani et al., 2016; Zhou et al., 2014; Tian et al., 2017; Char et al., 2019b; Di et al., 2019; Lee et al., 2019; Liang et al., 2019; Ren et al., 2019). This study aimed to investigate the function of the SlMAPK6 gene in the development of axillary buds in tomato plants; the CRISPR/Cas9 system was applied to generate SlMAPK6 gene knockout mutants. We investigated the development of axillary buds on CRISPR/Cas9-mediated slmapk3 mutants and wild-type (WT) plants as well as the possible by SIMAPK6-mediated regulatory mechanism that influences the development of axillary buds. This study provides insight into the regulatory mechanism of SIMAPK6-mediated axillary bud development in tomato plants.

\section{Materials and Methods}

\subsection{Plant Materials and Growth Conditions}

Dwarf tomato plants (Solanum lycopersicum) were used in this study as WT. The plants were grown in a pot (18 $\mathrm{cm} \times 22 \mathrm{~cm}$ ) with matrix (Pere matrix Technology Development Co., LTD, Jiangsu of China), and all plants were growth in artificial climate chamber and watered every three days with Hongland nutrient solution. Transgenic cultures were grown under controlled conditions with a 16-h-day/8-h-night photoperiod, a temperature of $25^{\circ} \mathrm{C} / 18{ }^{\circ} \mathrm{C}$ (day/night), a relative humidity of $65-70 \%$, and a light intensity of $250 \mu \mathrm{mol} \mathrm{m}^{-2} \mathrm{~s}^{-1}$. The first generation (T0) of transgenic tomato originated from tissue culture, and the homozygous SlMAPK6 knockout lines were screened from the seedlings of the second generation (T1). The third generation (T2) of tomato homozygous lines were used in subsequent experiments.

\subsection{Measurement of Plant Architecture Parameters}

To study differences between the WT and SIMAPK6-knockout lines, we observed the plant morphological parameters in flower stage, including the stem diameter, number of true leaves, internode length, number of axillary buds and compound leaves from SIMAPK6-knockout lines and WT plants.

\subsection{Construction of the SIMAPK6 Knockout Vector}

Three target sequences of the SIMAPK6 gene from the CRISPR-GE web tool (http://skl.scau.edu.cn/) were used. The target sequences were introduced into three single-guide RNA (sgRNA) expression cassettes using overlapping PCR. These three sgRNA expression cassettes were then ligated into a pCAMBIA2301 vector. The SIMAPK6 knockout vector was constructed by the company of RexBiotech.

The diagram of the SIMAPK6 knockout vector was shown in Supplemental Figure S1.

\subsection{Plant Transformation}

Via the freeze-thaw method (Jyothishwaran et al., 2007), the confirmed pCAMBIA2301/2x35S:hSpCas9-Tnos/ AtU6-26::SlMAPK6 binary vector was transferred into Agrobacterium tumefaciens strain GV3101. Transgenic plants were subsequently obtained through the cotyledon transformation method of Agrobacterium mediation. Transgenic lines were screened on the basis of kanamycin resistance. After the regenerated buds rooted, the regenerated shoots were grown for one month and then planted in sterilized soil at $25 \pm 2{ }^{\circ} \mathrm{C}$ with a $16-\mathrm{h} / 8-\mathrm{h}$ (light/dark) photoperiod. 


\subsection{Extraction of RNA and cDNA Synthesis}

RNA was extracted from the leaf samples by using Total RNA Extraction Reagent from Novizan Biotechnology Company (Nanjing, China) following the manufacturer's protocol. The RNA quality was observed by nano photometry (Thermo Scientific, China). After extraction, RNA was transcribed into cDNA by using a HiScript II First Strand cDNA Synthesis Kit from Novizan Biotechnology Company (Nanjing, China) following the manufacturer's protocol, and the concentrations of the cDNAs were adjusted to be the same.

\subsection{Quantitative Real-Time PCR Analysis}

Quantitative RT-PCR (qRT-PCR) was performed using a CFX96 ${ }^{\mathrm{TM}}$ Real-Time System (C1000TM Thermal Cycler, Bio-Rad, USA). All reactions were performed using a SYBR Premix Ex Taq II kit (Takara, China) in a $20 \mu \mathrm{l}$ total sample volume $(2.0 \mu \mathrm{l}$ of cDNA, $10.0 \mu \mathrm{l}$ of $2 \times$ SYBR Premix Ex Taq, $1.8 \mu \mathrm{l}$ of primers, and $6.2 \mu \mathrm{l}$ of distilled deionized water). The qRT-PCR procedure was as follows: predenaturation at $95{ }^{\circ} \mathrm{C}$ for $10 \mathrm{~min}$, denaturation at $95{ }^{\circ} \mathrm{C}$ for $15 \mathrm{~s}$, and annealing at $60{ }^{\circ} \mathrm{C}$ for $15 \mathrm{~s}$, for a total of 40 cycles. Standard curves were run simultaneously, and three replications were used for each sample. Tomato $C A C$ (SlCAC) was used as an internal standard as previously described by Exposito-Rodriguez et al. (2008) and Guo et al. (2017). All the primers used for qRT-PCR are shown in Supplemental Table S1.

\section{Results}

\subsection{Transcription Levels of the SIMAPK6 in WT}

As Figure1 A shows, SIMAPK6 was expressed in all tissues we examined. Among these transcripts, SlMAPK6 transcripts were detectable at very high levels in the tissues of the stems, leaves, and flowers, but the transcripts showed relatively low expression in the fruits, compared to the root (Figure 1). The relative expression of sepals had not a significant difference compared to the root. These results indicated that the SIMAPK6 gene plays a vital role in leaf and stem development.

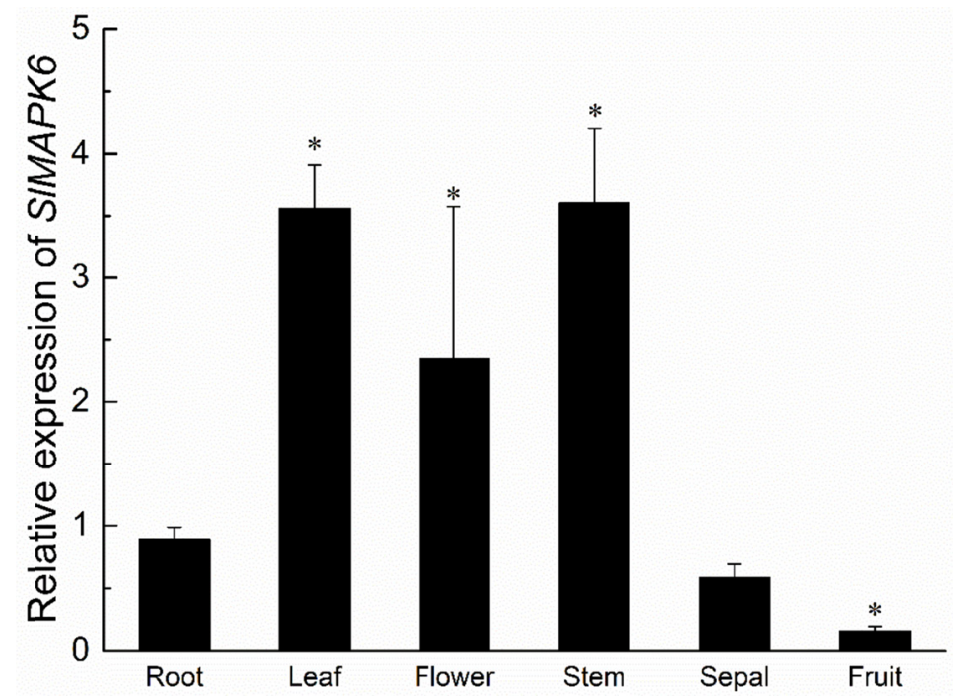

Figure 1. Transcriptional pattern analysis of SIMAPK6 in WT tomato plants

Note. Relative expression patterns of SIMAPK6 in different tissues and organs of WT plants. The data represent the means of three replicates with three biological repeats. The error bars indicate the SEs. The single asterisk (*) indicates significant difference.

\subsection{CRISPR/Cas9-Mediated Mutagenesis in SIMAPK6}

The three target sequences, Target1, Target 2 and Target3, those we selected were located in the third exon, which encodes the functional domain for the SIMAPK6 protein (Figure 2A). A schematic diagram of the recombinant vector, which was used to generate transgenic tomato plants, is depicted in Figure 2B. Through Agrobacterium-mediated transformation, kanamycin-resistant tomato plants were obtained. The results showed that two plants were edited successfully from 12 transgenic plants (T1), and the editing efficiency was $16.67 \%$. To investigate the functions of SIMAPK6, a CRISPR-Cas9 construct targeting SIMAPK6 (Figure 2B) was 
constructed, and several knockout lines were generated, which were confirmed by PCR with Cas9-specific primers. In addition, the target fragments, Target1, Target 2 and Target3, were sequenced and compared to the corresponding fragment in WT. Results shown that the target fragments had been changed, such as substitution, deletion, insertion (Figure 2C). So, the SIMAPK6-knockout lines, CRISPR-3 and CRISPR-7, had mutated successfully. The primers used for vector construction, positive transgenic plant detection and target fragments are shown in Supplemental Table S2. In addition, we also detected all possible off-target sites, and the results showed that none of the off-target sites changed after sequencing (Supplementary Table S3).

(A)

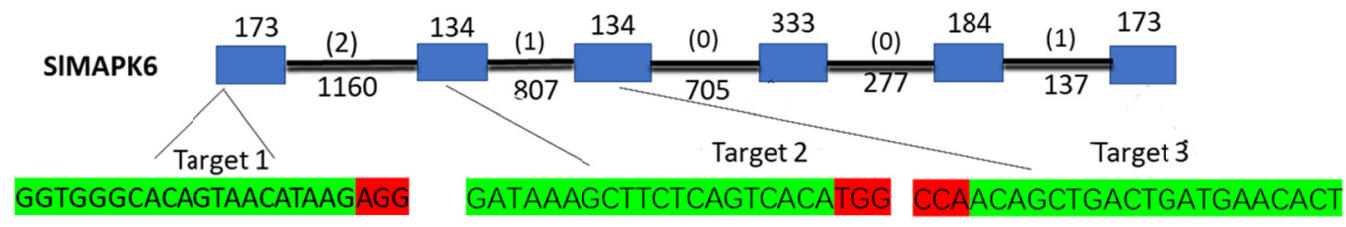

(B)

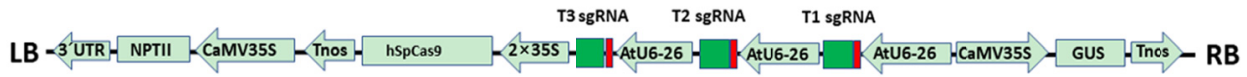

a

Editing type of SIMAPK6-target1 (T1)

(c) WT ATGAGTTGCAACCCCTCTTATGTTACTGTGCACGCCTTGCTACTCTGAACTTTCATCATGATACATCTCATGTGA
CRISPR-3 ATGAGTTGCAACCCCTCTTATGTTACTGTGCACGCCATGATCATCTGAACTTGCATCATGATACATCTCATGTGA (5bp substitution)
CRISPR-7 ATGAGTTGCAACCCCTCTTATGTTACTGTGCCCACCATGATCAGCTGAACTTGAATCATGAGACATCTCATGTGA (10bp substitution)
C...
CCTCTTATGTTACTGTGCCCACC.

b Editing type of SIMAPK6-target2 (T1)

WT CCGGCGCGCGCGCGAGACGCAAGAAGAGTATTGCTTTCCAAGTACACATGGATCATGAG

CRISPR-3 AAGGGGCACGGCCGGAGAAGCAAGAGAGTATAGCTTCTCA-GT-CACATGGATCATGAG (3bp substitution; 2bp deletion)*

CRISPR-7 GCGGAGCGGGCG.GAGAAGCAAGAAGAGAATTGCTTTCTAAGT-CACATGGATCATGAG (2bp substitution; 1bp deletion; 1bp ins ertion) GATAAAGCTTCTCAGTCACATGG. (Target2)

c

Editing type of SIMAPK6-target3 (T1)

WT TAGCTTACTCGACAGTGTTCTTTGGTCAGCTGITGGTTGGAATGAATAATCTGATGAAG CRISPR-3 TAGCTTACTCGACAGTGTTCTTTGGTCAGCTGTIGGTTGGAATGAATAATCTGATGAAG (WT)

CRISPR-7 TAGCTTACTCGACAGTGTGTATCGTTCAGCTGTIGGTTGGAATGAATAATCTGATGAAG (5bp substitution) AGTGTTCATCAGTCAGCTGTTGG (Target3)

Figure 2. Schematic diagram of the SIMAPK6 gene structure and vector construction of the CRISPR/Cas9 system

Note. (A) Schematic diagram of the SIMAPK6 gene structure and target sequences. The black lines indicate introns, and the blue rectangles indicate exons. The protospacer adjacent motif (PAM) sequence is shown with a red background, and the target sequence is marked with a green background. (B) Vector construction of the CRISPR/Cas9 system. NPTII, kanamycin resistance gene; CaMV35S, the promoter of the $35 \mathrm{~S}$ cauliflower mosaic virus; Tnos, gene terminator; AtU6-26, Arabidopsis U6-26 promoter. (C) CRISPR/Cas9-mediated mutation sequence of the tomato SIMAPK6 gene. Representative genotypes of slmapk6 mutants. Target sequences are shown with a green background, with PAM sequences shown with a red background. Deletions are shown by a black dash. Substitutions are shown with a purple background, and insertions are expressed with a yellow background.

\subsection{Knockout of SlMAPK6 Alters Plant Morphogenesis}

Two SIMAPK6-knockout lines (CRISPR-3 and CRISPR-7) were chosen and used for a more detailed phenotypic and molecular analysis based on their expression level of SIMAPK6.

The two SlMAPK6-knockout lines (CRISPR-3 and CRISPR-7) were different from the WT plants within a few days after germination (Figure 3A). Compared with the WT lines, the two knockout lines (CRISPR-3 and CRISPR-7) exhibited increased plant height and larger compound leaves (Figure 4B). The increase in adventitious roots in the knockout lines was more prominent than that in the WT (Figure 3G). 


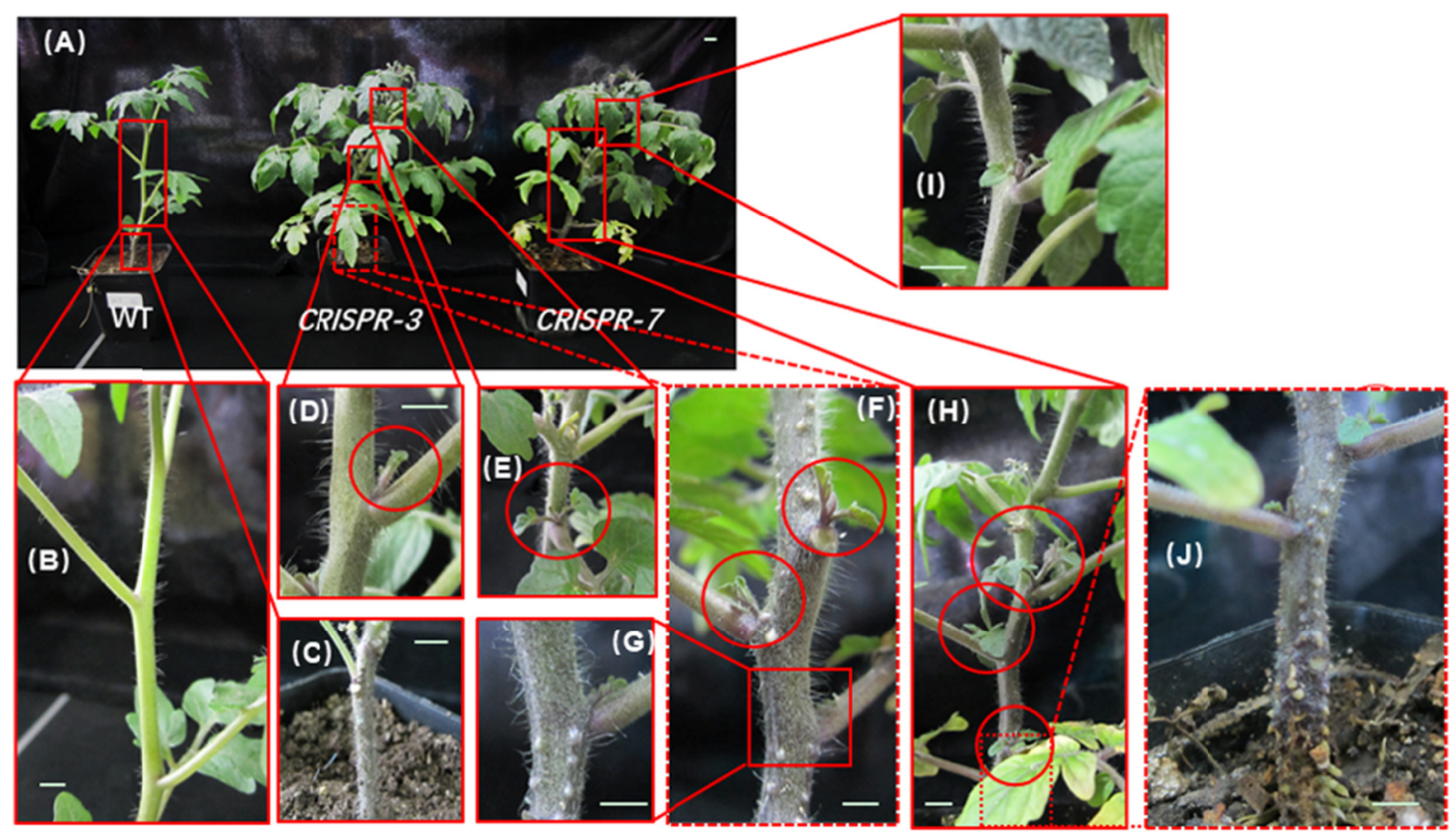

Figure 3. Knockout of SIMAPK6 alter the plant morphology

Note. (A)-(J) represent the morphology of SIMAPK6 knockout lines (CRISPR-3 and CRISPR-7) and WT plants.
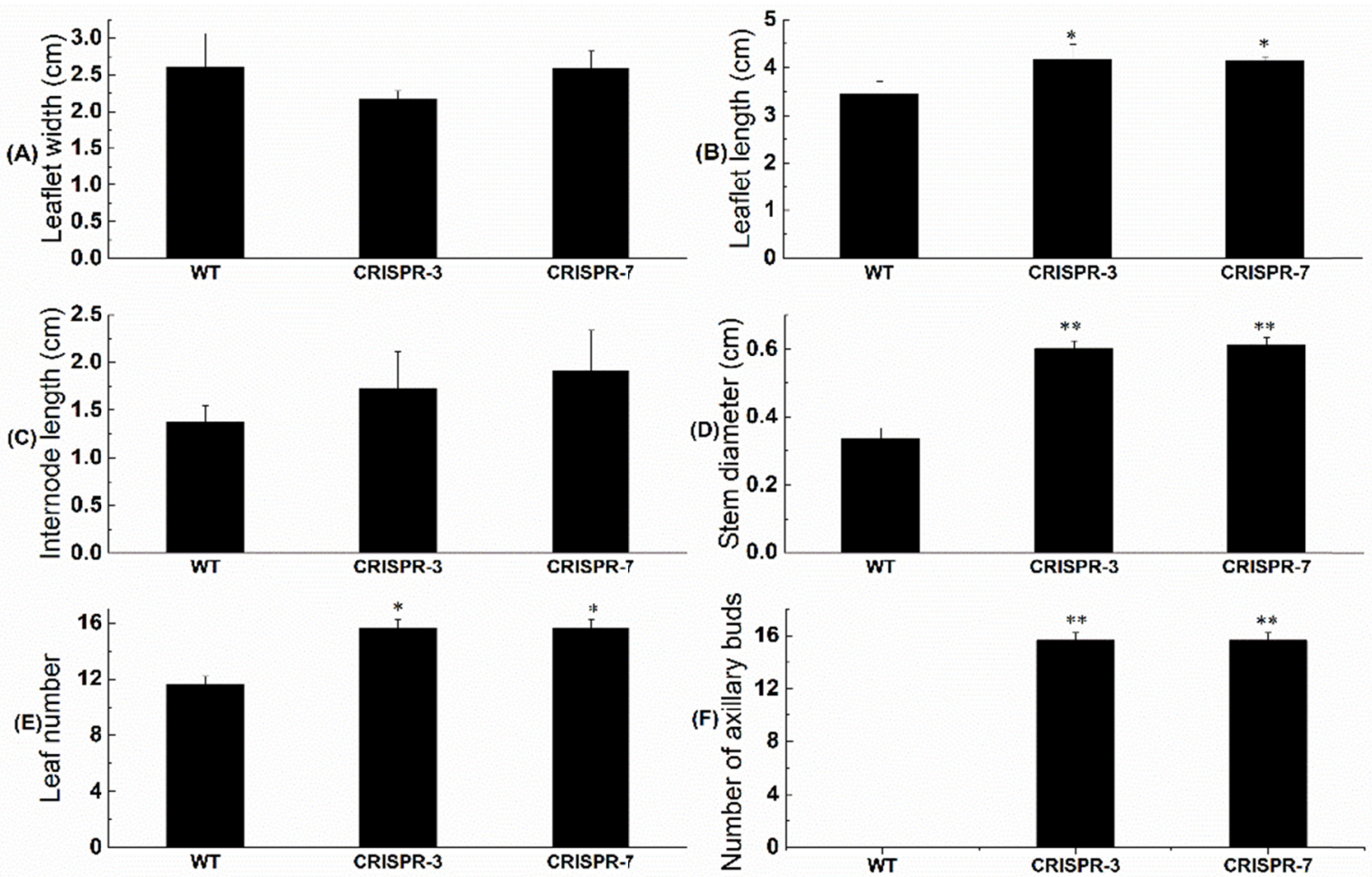

Figure 4. Data related to plant morphology in SIMAPK6 knockout lines (CRISPR-3 and CRISPR-7) and WT plants

Note. (A)-(F) represent the leaflet width, leaf length, internode length, stem diameter, leaf number and number of axillary buds in SIMAPK6-knockout lines and WT. Each value represents the mean $\pm \mathrm{SE}$ of three replicates. The asterisks indicate significant differences $(P<0.05)$ between the WT and SIMAPK6-knockout lines. 
3.4 Expression Analysis of the Genes Involved in GA Biosynthesis, the GA Response and Cell Elongation in WT and SIMAPK6-Knockout Lines

In SIMAPK6-knockout lines, the relative expression levels of GA20ox3 and GA3ox1 were significantly low (Figures 5A and 5B), which might lead to the reduction in GA content in the SIMAPK6-knockout lines, CRISPR-3 and CRISPR-7. Furthermore, GAI, a repressor of GA responses, was assessed at the transcriptional level. It was found that the expression level of GAI was significantly higher in the SIMAPK6-knockout lines (Figure 5C).

Members of the PRE (Paclobutrazol Resistance) family positively regulate cell elongation, and they encode protein structures that have a small helix-loop-helix (HLH) motif (Lee et al., 2006; Zhang et al., 2009). Three tomato PREs, PRE1, PRE2 and PRE3, were identified, and their transcription was examined. The results showed that the expression levels of PRE1, PRE2 and PRE3 were significantly lower in the knockout lines (Figures $5 \mathrm{C}-5 \mathrm{E})$.

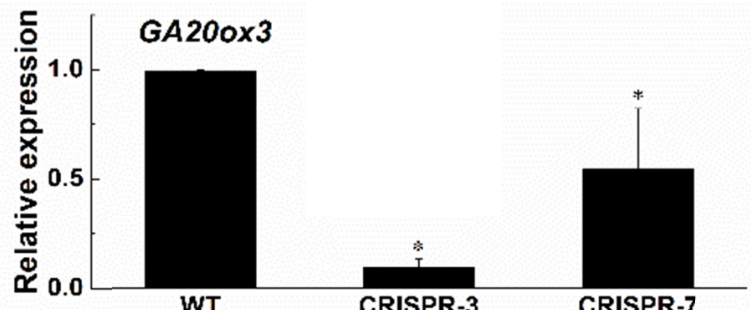

(A)

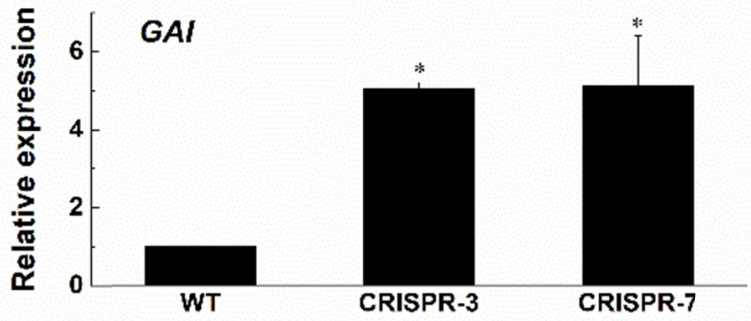

(C)

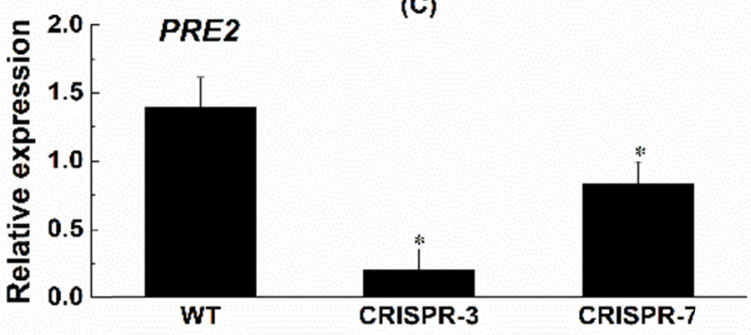

(E)

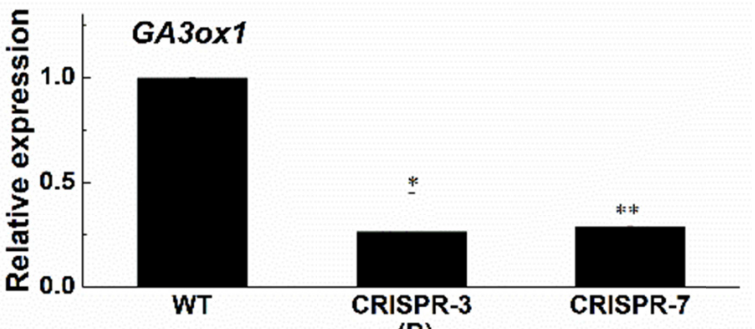

(B)

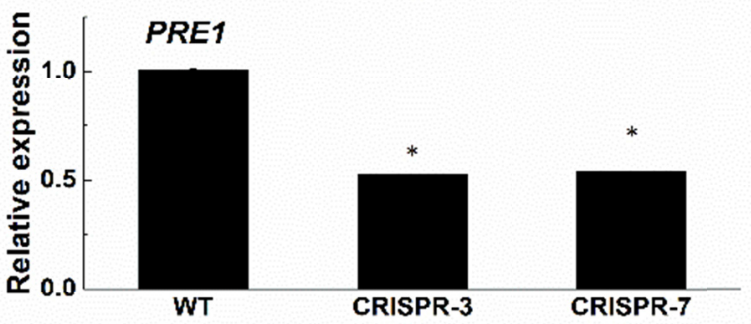

(D)

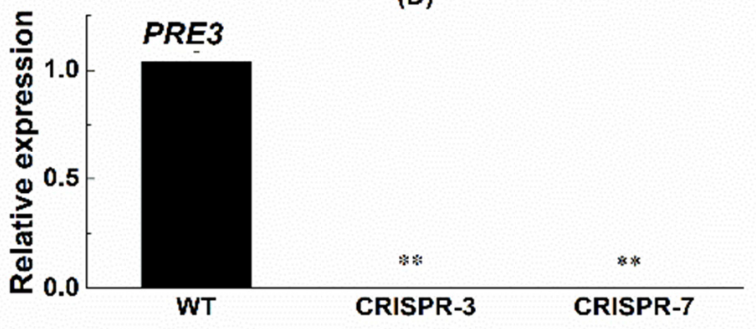

(F)

Figure 5. Expression levels of genes involved in the GA response, GA biosynthesis and cell elongation in WT and SIMAPK6-knockout lines

Note. (A)-(C) show the relative transcript levels of GA3ox1, GA20ox3 and GAI, respectively, in WT and SIMAPK6-knockout lines. (D)-(F) show the transcript levels of PRE1, PRE2 and PRE3, respectively, in WT and SIMAPK6-knockout lines. Each data from three biological and technological replicates and represents the mean \pm SE. The single asterisks $(*)$ indicate significant differences $(P<0.05)$ and double asterisks $(* *)$ indicate highly significant differences $(P<0.01)$.

\subsection{Expression of Tomato Strigolactone Biosynthesis- and Lateral Bud Development-Related Genes in SlMAPK6-Knockout Lines}

Our results showed that $S I C C D 7$ and $S I C C D 8$ were significantly upregulated in the lateral buds of the knockout plants (Figures 6A and 6B). In addition, the expression of three lateral bud development-related genes, Lateral suppressor (Ls) (Schumacher et al., 1999), Blind (BL) (Schmitz et al., 2002) and BRC1b/TCP8 (Martín - Trillo et al., 2011), was examined in WT and two SIMAPK6-knockout lines. The transcript level of tomato $L s$, encoding a new member of the VHIID protein, did not differ between the knockout lines and the WT (Figure 6C). 
The $B L$ gene can regulate the formation of lateral meristems by encoding a MYB transcription factor (Schmitz et al., 2002). The BRClb gene is involved in the control of cell growth and proliferation in lateral organs and meristems by encoding a transcription factor of the TCP family (Proliferating Cell Factors, Cycloidea, Teosinte Branched 1) (Cubas et al., 1999; Martín-Trillo et al., 2010). Significantly decreased expression levels of these two genes were found in the lateral buds of transgenic plants (Figures 6D and 6E).

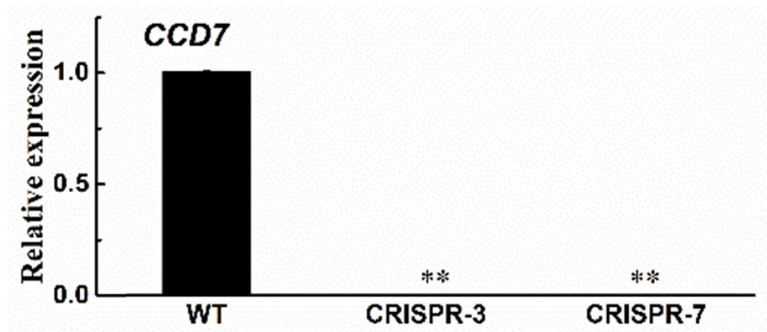

(A)

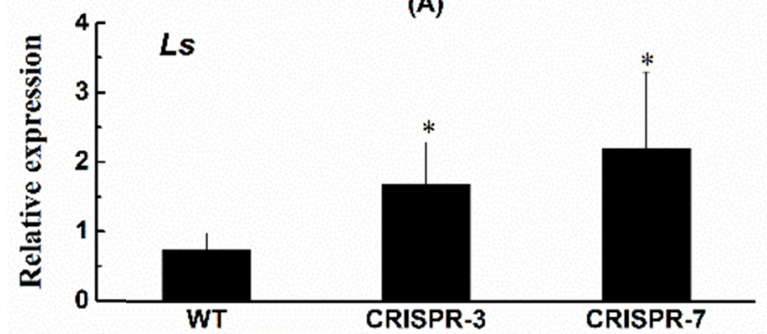

(C)

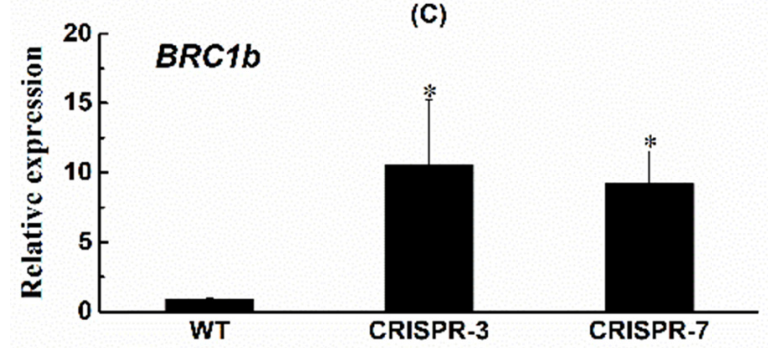

(E)

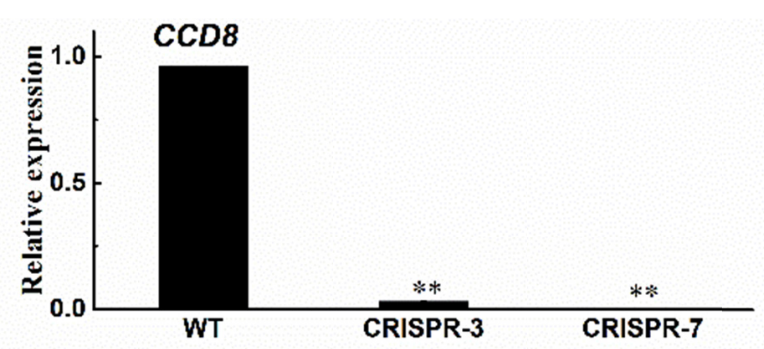

(B)

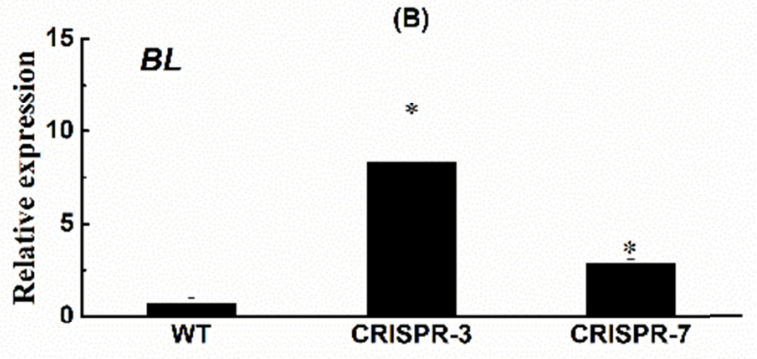

(D)

Figure 6. Relative expression levels of tomato lateral bud development- and strigolactone biosynthesis-related genes in WT and SIMAPK6-knockout lines

Note. (A) and (B) show the relative expression levels of strigolactone biosynthesis genes $C C D 7$ and $C C D 8$, respectively, in lateral buds of the WT and the SIMAPK6-knockout lines. (C), (D) and (E) show the relative expression of the lateral bud development-related genes $L s, B L$ and $R B C 1 b$, respectively, in the lateral buds of the WT and SIMAPK6-knockout lines. Each data from three biological and technological replicates and represents the mean $\pm \mathrm{SE}$ of. The single asterisks $(*)$ indicate significant differences $(P<0.05)$ and the double asterisks $(* *)$ indicate highly significant differences $(P<0.01)$.

\section{Discussion}

In this study, a gene-editing technology was used to knockout the gene of SIMAPK6 to explored its function. It was found that the SIMAPK6-knockout line, CRISPR-3 and CRISPR-7 have special phenotype, for example, an increased number of axillary buds and an enhancement in the length of leaflets, stem diameter, number of axillary buds and number of compound leaves. These results suggest that SIMAPK6 plays a key role in maintaining plant growth and morphogenesis.

To determine the molecular mechanism underlying axillary buds in the SIMAPK6-knockout lines, cell elongation, GA biosynthesis and GA response-related genes in tomato, including PRE1, PRE2, PRE3, GA20ox3, GA3ox1 and GAI, were observed. Among them, the expression levels of GA20ox3 and GA3oxl were slightly downregulated in knockout plant leaves, which may be involved in the production of a minimum amount of GAs in SIMAPK6-knockout lines. However, GAI, a repressor of GA responses, was highly upregulated in the SIMAPK6-knockout lines. These results suggest that the knockout of SlMAPK6 affects the biosynthesis and 
catabolism of GA. Our results are in accordance with those of a previous study of Martín-Trillo et al. (2010), where it was shown that the expression level of PRE1 was regulated by GA. Moreover, the expression levels of PRE1, PRE2 and PRE3 were significantly reduced in SIMAPK6-knockout lines (Martín-Trillo et al., 2010). However, our results suggest that SIMAPK6 positively regulates GA-related genes. Our results are in agreement with the development of tomato axillary buds being regulated by members of several transcription factor families, such as MYBs (e.g., the BL gene) (Schmitz et al., 2002), TCPs (e.g., the BRClb gene) (Cubas et al., 1999; Martín-Trillo et al., 2010) and GRASs (e.g., the Ls gene) (Schumacher et al., 1999).

During the vegetative growth of the plants whose SIMAPK6 gene was knocked out, there were a greater number of compound leaves and more vigorous plants. These results showed an increase in the vegetative growth of the SIMAPK6-knockout lines. These results are in agreement with those of a previous study in which SlCCD7 and SICCD 8 were reported to inhibit the growth of branches of tomato plants through the methods of expressing antisense constructs or RNAi-mediated silencing (Kohlen et al., 2012; Vogel et al., 2010). The expression levels of the $S l C C D 7$ and SlCCD 8 genes were also examined in the SIMAPK6-knockout lines. It was observed that the expression levels of both genes were downregulated in SIMAPK6-knockout plants, indicating that with the abundant production of axillary buds, strigolactone biosynthesis might be decreased.

In this study, the increase in the number of axillary buds was examined due to the loss of function of SIMAPK6 in the knockout lines. The level of expression was observed in lateral buds for related developmental genes, such as $L s, B L$ and $B R C 1 b$, in both WT and knockout plants. The highly expressed $B L$ genes in the SlMAPK6 knockout lines indicated that SIMAPK6 negatively regulates $B L$ genes. Moreover, the $B R C 1 b$ gene were highly upregulated in the SIMAPK6 knockout lines, which contradicts the increased axillary bud phenotype of the knockout plants. Shoot branching of angiosperms determines the aspects of plant morphological structure, such as visibility to pollinators, plant height and nutrient allocation. Thus, the vigorous vegetative growth of the SIMAPK6 knockout plant probably accounts for the low fruit set percentage of the transgenic lines.

These results were also in agreement with those of a previous study in which RNA interference was applied to the $B L$ control of the development of lateral axes. After interference with $B L$ in tomato, the number of lateral axes decreased because the initiation of lateral meristems was stopped (Schmitz et al., 2002). SlBRC1b was found to suppress the growth of shoot branches because increased branch outgrowth was shown in SlBRC1b-loss-of-function tomato plants (Martín-Trillo et al., 2011).

\section{Conclusions}

The function of SIMAPK6 was studied through CRISPR-Cas9 technology. First, it was observed that SIMAPK6 was ubiquitously expressed in the different tissues we examined, and SIMAPK6 expression levels were higher in the tissues of the leaves, stems and flowers than in the tissues of the roots, fruits and sepals. Second, the SIMAPK6-knockout lines CRISPR-3 and CRISPR-7 were obtained using CRISPR-Cas9 technology. It was found that, as a negative regulator, SIMAPK6 plays a key role in regulating the development of axillary buds and architecture of tomato plants. In addition, a potential molecular mechanism for increasing the number of axillary buds was studied in SIMAPK6-knockout plants. It was possible that SIMAPK6 negatively regulate strigolactone biosynthesis via $S l C C D 7$ and SlCCD8 and positively regulated GA biosynthesis via PRE1, PRE2, PRE3, GA20ox3, GA3oxl and GAI, which led to the phenotype of increased numbers of axillary buds.

\section{Acknowledgments}

This work was supported by the National Natural Science Foundation of China (No. 32060679; No. 31760594; No. 31960604), Research Project on Talent Introduction of Guizhou University (No. [2017]50) and National Natural Fund Cultivation Project of Guizhou University (No. [2017] 5788-28).

\section{References}

Char, S. N., Li, R., \& Yang, B. (2019a). CRISPR/Cas9 for Mutagenesis in Rice. Methods in Molecular Biology, 1864, 279-293. https://doi.org/10.1007/978-1-4939-8778-8_19

Char, S. N., Wei, J., Mu, Q., Li, X., Zhang, Z. J., Yu, J., \& Yang, B. (2019b). An Agrobacterium-delivered CRISPR/Cas9 system for targeted mutagenesis in sorghum. Plant Biotechnology Journal, 18(2), 1-3. https://doi.org/10.1111/pbi.13229

Chen, X. J., Xia, X. J., Guo, X., Zhou, Y. H., Shi, K., Zhou, J., \& Yu, J. Q. (2016). Apoplastic $\mathrm{H}_{2} \mathrm{O}_{2}$ plays a critical role in axillary bud outgrowth by altering auxin and cytokinin homeostasis in tomato plants. New Phytologist, 211, 1266-1278. https://doi.org/10.1111/nph.14015 
Cubas, P., Lauter, N., Doebley, J., \& Coen, E. (1999). The TCP domain: A motif found in proteins regulating plant growth and development. Plant Journal, 18, 215-222. https://doi.org/10.1046/j.1365-313X.1999. 00444.X

Di, Y. H., Sun, X. J., Hu, Z., Jiang, Q. Y., Song, G. H., Zhang, B., ... Zhang, H. (2019). Enhancing the CRISPR/Cas9 system based on multiple GmU6 promoters in soybean. Biochemical and Biophysical Research Communications, 519, 819-823. https://doi.org/10.1016/j.bbrc.2019.09.074

Exposito-Rodriguez, M., Borges, A. A., Borges-Perez, A., \& Perez, J. A. (2008). Selection of internal control genes for quantitative real-time RT-PCR studies during tomato development process. BMC Plant Biology, 8(1), 131-143. https://doi.org/10.1186/1471-2229-8-131

Feng, Z., Mao, Y., Xu, N., Zhang, B., Wei, P., Yang, D. L., ... Zhu, J. K. (2014). Multigeneration analysis reveals the inheritance, specificity, and patterns of CRISPR/Cas-induced gene modifications in Arabidopsis. Proceedings of the National Academy of Sciences, 111(12), 4632-4637. https://doi.org/10.1073/pnas.14008 22111

Group, M., Ichimura, K., Shinozaki, K., Tena, G., Sheen, J., Henry, Y., ... Walker, J. C. (2002). Mitogen-activated protein kinase cascades in plants: A new nomenclature. Trends in Plant Science, 7, 301-308. https://doi.org/10.1016/S1360-1385(02)02302-6

Guo, X. H., Chen, G. P., Naeem, M., Yu, X. H., \& Tang, B. Y. (2017). The MADS-box gene SlMBP11 regulates plant architecture and affects reproductive development in tomato plants. Plant Science, 258, 91-101. https://doi.org/10.1016/j.plantsci.2017.02.005

Huh, Y. J., Han, B. H., Park, S. K., Lee, S. Y., Kil, M. J., \& Pak, C. H. (2013). Inhibition of chrysanthemum axillary buds via transformation with the antisense tomato lateral suppressor gene is season dependent. Horticulture Environment and Biotechnology, 54, 280-287. https://doi.org/10.1007/s13580-013-0116-9

Ito, Y., Nishizawa-Yokoi, A., Endo, M., Mikami, M., \& Toki, S. (2015). CRISPR/Cas9-mediated mutagenesis of the RIN locus that regulates tomato fruit ripening. Biochemical and Biophysical Research Communications, 467(1), 76-82. https://doi.org/10.1016/j.bbrc.2015.09.117

Jyothishwaran, G., Kotresha, D., Selvaraj, T., Srideshikan, S., Rajvanshi, P., \& Jayabaskaran, C. (2007). A modified freeze-thaw method for efficient transformation of Agrobacterium tumefaciens. Current Science, 93(6), 770-772. Retrieved from http://www.jstor.org/stable/24099118

Kohlen, W., Charnikhova, T., Lammers, M., Pollina, T., Toth, P., Haider, I., ... Lopez-Raez, J. A. (2012). The tomato CAROTENOID CLEAVAGE DIOXYGENASE8 (SICCD8) regulates rhizosphere signaling, plant architecture and affects reproductive development through strigolactone biosynthesis. New Phytologist, 196, 535-547. https://doi.org/10.1111/j.1469-8137.2012.04265.x

Kong, F. L., Wang, J., Cheng, L., Liu, S. Y., Wu, J., Peng, Z., \& Lu, G. (2012). Genome-wide analysis of the mitogen-activated protein kinase gene family in Solanum lycopersicum. Gene, 499(1), 108-120. https://doi.org/10.1016/j.gene.2012.01.048

Lee, K., Zhang, Y., Kleinstiver, B. P., Guo, J. A., Aryee, M. J., Miller, J., ... Wang, K. (2019). Activities and specificities of CRISPR/Cas9 and Cas12a nucleases for targeted mutagenesis in maize. Plant Biotechnology Journal, 17, 362-372. https://doi.org/10.1111/pbi.12982

Lee, S., Lee, S., Yang, K. Y., Kim, Y. M., Park, S. Y., Kim, S. Y., \& Soh, M. S. (2006). Overexpression of PRE1 and its homologous genes activates Gibberellin-dependent responses in Arabidopsis thaliana. Plant Cell Physiology, 47(5), 591-600. https://doi.org/10.1093/pcp/pcj026

Liang, Z., Chen, K., \& Gao, C. (2019). Biolistic Delivery of CRISPR/Cas9 with Ribonucleoprotein Complex in Wheat. Methods in Molecular Biology, 1917, 327-335. https://doi.org/10.1007/978-1-4939-8991-1_24

Lv, X. Z., Ge, S. B., Jalal Ahammed, G., Xiang, X., Guo, Z. X., Yu, J. Q., \& Zhou, Y. H. (2017). Crosstalk between Nitric Oxide and MPK1/2 Mediates Cold Acclimation-induced Chilling Tolerance in Tomato. Plant and Cell Physiology, 58, 1963-1975. https://doi.org/10.1093/pcp/pcx134

Martín-Trillo, M., \& Cubas, P. (2010). TCP genes: A family snapshot ten years later. Trends in Plant Science, 15(1), 31-39. https://doi.org/10.1016/j.tplants.2009.11.003

Martín-Trillo, M., Grandío, E. G., Serra, F., Marcel, F., Rodríguez-Buey, M. L., Schmitz, G., ... Cubas, P. (2011). Role of tomato BRANCHED1-like genes in the control of shoot branching. Plant Journal, 67(4), 701-714. https://doi.org/10.1111/j.1365-313X.2011.04629.x 
Nishitani, C., Hirai, N., Komori, S., Wada, M., Okada, K., Osakabe, K., ... Osakabe, Y. (2016). Efficient Genome Editing in Apple Using a CRISPR/Cas9 system. Scientific Reports, 6, 31481-31489. https://doi.org/10.1038/srep31481

Reem, N. T., \& Van Eck, J. (2019). Application of CRISPR/Cas9-Mediated Gene Editing in Tomato. Methods in Molecular Biology, 1917, 171-182. https://doi.org/10.1007/978-1-4939-8991-1_13

Ren, F., Ren, C., Zhang, Z., Duan, W., Lecourieux, D., Li, S., \& Liang, Z. (2019). Efficiency Optimization of CRISPR/Cas9-Mediated Targeted Mutagenesis in Grape. Front. Plant Science, 10, 612. https://doi.org/ 10.3389/fpls.2019.00612

Schmitz, G., Tillmann, E., Carriero, F., Fiore, C., Cellini, F., \& Theres, K. (2002). The tomato Blind gene encodes a $M Y B$ transcription factor that controls the formation of lateral meristems. Proceedings of the National Academy of Sciences, 99(2), 1064-1069. https://doi.org/10.1073/pnas.022516199

Schumacher, K., Schmitt, T., Rossberg, M., Schmitz, G., \& Theres, K. (1999). The Lateral suppressor (Ls) gene of tomato encodes a new member of the VHIID protein family. Proceedings of the National Academy of Sciences, 96, (1), 290-295. https://doi.org/10.1073/pnas.96.1.290

Smekalova, V., Doskocilova, A., Komis, G., \& Samaj, J. (2014). Crosstalk between secondary messengers, hormones and MAPK modules during abiotic stress signalling in plants. Biotechnology Advances, 32, 2-11. https://doi.org/10.1016/j.biotechadv.2013.07.009

Stulemeijer, I. J., Stratmann, J. W., \& Joosten, M. H. (2007). Tomato mitogen-activated protein kinases LeMPK1, LeMPK2, and LeMPK3 are activated during the $C f$-4/Avr4-induced hypersensitive response and have distinct phosphorylation specificities. Plant Physiology, 144(3), 1481-1494. https://doi.org/10.1104/ pp.107.101063

Tian, S., Jiang, L., Gao, Q., Zhang, J., Zong, M., Zhang, H., ... Xu, Y. (2017). Efficient CRISPR/Cas9-based gene knockout in watermelon. Plant Cell Reports, 36(3), 399-406. https://doi.org/10.1007/s00299016-2089-5

Vogel, J. T., Walter, M. H., Giavalisco, P., Lytovchenko, A., Kohlen, W., Charnikhova, T., ... Klee, H. J. (2010). SlCCD7 controls strigolactone biosynthesis, shoot branching and mycorrhiza-induced apocarotenoid formation in tomato. Plant Journal, 61(2), 300-311. https://doi.org/10.1111/j.1365-313X.2009.04056.X

Wang, F., Wang, C., Liu, P., Lei, C., Hao, W., Gao, Y., ... Zhao, K. (2016). Enhanced Rice Blast Resistance by CRISPR/Cas9-Targeted Mutagenesis of the ERF Transcription Factor Gene OsERF922. PLoS One, 11, 1-18. https://doi.org/10.1371/journal.pone.0154027

Widmann, C., Gibson, S., Jarpe, M. B., \& Johnson, G. L. (1999). Mitogen-Activated Protein Kinase: Conservation of a Three-Kinase Module from Yeast to Human. Physiological Reviews, 79(1), 143-180. https://doi.org/10.1152/physrev.1999.79.1.143

$\mathrm{Xu}$, J., \& Zhang, S. (2015). Mitogen-activated protein kinase cascades in signaling plant growth and development. Trends in Plant Science, 20(1), 56-64. https://doi.org/10.1016/j.tplants.2014.10.001

Yamaguchi, S. (2008). Gibberellin metabolism and its regulation. Annual Review of Plant Biology, 59(1), 225-251. https://doi.org/10.1007/s003440010039

Zhang, L. Y., Bai, M. Y., Wu, J. X., Zhu, J. Y., Wang, H., Zhang, Z. G., ... Wang, Z. Y. (2009). Antagonistic HLH/bHLH Transcription Factors Mediate Brassinosteroid Regulation of Cell Elongation and Plant Development in Rice and Arabidopsis. The Plant Cell, 21(12), 3767-3780. https://doi.org/10.1105/ tpc.109.070441

Zhou, J., Xia, X. J., Zhou, Y. H., Shi, K., Chen, Z. X., \& Yu, J. Q. (2014). RBOH1-dependent $\mathrm{H}_{2} \mathrm{O}_{2}$ production and subsequent activation of MPK1/2 play an important role in acclimation-induced cross-tolerance in tomato. Journal of Experimental Botany, 65(2), 595-607. https://doi.org/10.1093/jxb/ert404

Zhou, X., Zha, M., Huang, J., Li, L., Imran, M., \& Zhang, C. (2017). StMYB44 negatively regulates phosphate transport by suppressing expression of PHOSPHATE1 in potato. Journal of Experimental Botany, 68(5), 1265-1281. https://doi.org/10.1093/jxb/erx026 


\section{Supplementary}

Supplemental Table S1. PCR primers used in this study

\begin{tabular}{|c|c|}
\hline Primers & Primer sequences $\left(5^{\prime} \rightarrow 3^{\prime}\right)$ \\
\hline SIMAPK6-F & CGTTCCAGTTCAAAAGTTCTCACATGATCA \\
\hline SIMAPK6-R & CCACCATATTTATTCAGTCATCTTCACTGGG \\
\hline Target1-F & CAAGATTCTTCAACСССТTTTCT \\
\hline Target1-R & CACGACCAATAGGTCTGATAGGA \\
\hline Target2-F & GAAGATTGGGAATGCATTTGATA \\
\hline Target2-R & AACAAGGCAAAGACATTTGAAGA \\
\hline Target3-F & ATGTTATACGGCCTCCTCAAAAG \\
\hline Target3-R & CCTGAAAAGTGTTTACCGCTATG \\
\hline Kan-F & GGCGATACCGTAAAGCACGA \\
\hline Kan-R & ATGGATTGCACGCAGGTTCT \\
\hline SIMAPK6-Q-F & TGGAGATTTTGGGCTAGCAA \\
\hline SIMAPK6-Q-R & CCAGGAAAAAGGGGTTGTCT \\
\hline$S I C A C-F$ & CCTCCGTTGTGATGTAACTGG \\
\hline$S I C A C-R$ & ATTGGTGGAAAGTAACATCATCG \\
\hline GA20ox3 -Q-F & AGCCAAATTATGCTAGTGTTAC \\
\hline GA20ox3 -Q-R & TTTTATGAGATTTGTGTCAACC \\
\hline GA3oxl-Q-F & ATAGGCACCCACCCTTGTATA \\
\hline GA3oxl-Q-R & GGATGAAAGTGCCTTGTCAAAAT \\
\hline GAI-Q-F & CCAGCACTTGTCATTCTTACCC \\
\hline GAI-Q-R & AAAGCTCATCCATTCCAGCA \\
\hline PRE1-Q-F & CGAAAGAACGAAAAGAGAGACATT \\
\hline PRE1-Q-R & GCTAGAGCGACGATTGCGAA \\
\hline PRE2-Q-F & TATGTCTGGGAGAAGGTCAAGGA \\
\hline PRE2-Q-R & CGACGATTACGAATTTCAGGAAG \\
\hline PRE3-Q-F & TTCACACTCTCCATAGCAACACAT \\
\hline PRE3-Q-R & TCСТCACTTATCСТTGATCCTCC \\
\hline$C C D 7-\mathrm{Q}-\mathrm{F}$ & AGCCAAGAATTCGAGATCCC \\
\hline$C C D 7-\mathrm{Q}-\mathrm{R}$ & GGAGAAAGCCCACATACTGC \\
\hline$C C D 8-\mathrm{Q}-\mathrm{F}$ & CCAATTGCCTGTAATAGTTCC \\
\hline$C C D 8-\mathrm{Q}-\mathrm{R}$ & GCCTTCAACGACGAGTTCTC \\
\hline$L s-\mathrm{Q}-\mathrm{F}$ & TGTTTTCTACCTCCACCGCCT \\
\hline$L s-\mathrm{Q}-\mathrm{R}$ & ATGATTTGCTTCCTTCTCCGC \\
\hline$B L-\mathrm{Q}-\mathrm{F}$ & GGAAGATTTGATAGTTGTGTTG \\
\hline$B L-\mathrm{Q}-\mathrm{R}$ & CAAAAATAGAGCTACACAAAACC \\
\hline$B R C 1 b-\mathrm{Q}-\mathrm{F}$ & AGAAGGCCGAGGCGAAAA \\
\hline$B R C 1 b-\mathrm{Q}-\mathrm{R}$ & GACCACGAGCGGTGTTGATC \\
\hline$R b c S 3 B-\mathrm{Q}-\mathrm{F}$ & TGCTCAGCGAAATTGAGTACCTAT \\
\hline$R b c S 3 B-\mathrm{Q}-\mathrm{R}$ & AACTTCCACATGGTCCAGTATCTG \\
\hline
\end{tabular}




\section{Supplemental Table S2. Primers used for vector construction}

\begin{tabular}{|c|c|c|c|}
\hline Aim & Primer name & Primer sequence & Product size (bp) \\
\hline \multirow[t]{2}{*}{ PCR of potential off-target 1 for target sequence 1} & Off-target1.1-F & TCACTCCTTGGCCTTTTCTT & 240 \\
\hline & Off-target1.1-R & GGACATGCATGCTTATCTTGAA & \\
\hline \multirow[t]{2}{*}{ PCR of potential off-target 2 for target sequence 1} & Off-target1.2-F & CCATGATGTGTGGGATCTTCT & 238 \\
\hline & Off-target1.2-R & CAGGACAGTGACCATCCCTAA & \\
\hline \multirow[t]{2}{*}{ PCR of potential off-target 1 for target sequence 2} & Off-target2.1-F & GCTCGAGTTTGTTCCGAATC & 222 \\
\hline & Off-target2.1-R & CACTTCCTCCTGAACCCTGA & \\
\hline \multirow[t]{2}{*}{ PCR of potential off-target 2 for target sequence 2} & Off-target $2.2-\mathrm{F}$ & TTTTTCTTCAGTGCCGCTTT & 196 \\
\hline & Off-target2.2-R & TGCACAAGGTACAAGGCAGT & \\
\hline \multirow{2}{*}{ PCR of potential off-target 1 for target sequence 3} & Off-target3.1-F & GGCCACCACAAACTGAGAAT & 227 \\
\hline & Off-target3.1-R & TGGCTAGTTCAGGCGACATA & \\
\hline \multirow[t]{2}{*}{ PCR of potential off-target 2 for target sequence 3} & Off-target3.2-F & TGCTTTCACATTGCCAGGTA & 241 \\
\hline & Off-target3.2-R & CAGGCACTGCATTACCATCA & \\
\hline
\end{tabular}

Supplementary Table S3. Mutations of potential miss sequences

\begin{tabular}{lllll}
\hline Target & Name of potential off-target sites & Potential off-target sequence & No. mismatch bases & No. plants tested \\
\hline T1 & T1-O1 & To. mutations \\
\hline T2 & T2-O1 & GGTGTGAACAGTAACATGAGGAG & 3 & 30 \\
T2-O2 & CAAATAGCTTATCAGTCACAGGG & 4 \\
T3 & T3-O1 & GATAAAGCTTCTTCGTCACATGG & 2 \\
& T3-O2 & AGTGATCATCAGTCAACTGTTGG & 2 & 30 \\
& T3-O3 & AGGTCTCATAAGTCAGCTGTTGG & 4 & 30 \\
\end{tabular}

Note. The PAM sequence (NGG) is underlined, and the NAG analog was also used for testing. Mismatch bases are marked in red.

\section{Supplemental Figure S1. The diagram of SIMAPK6-knockout vector}

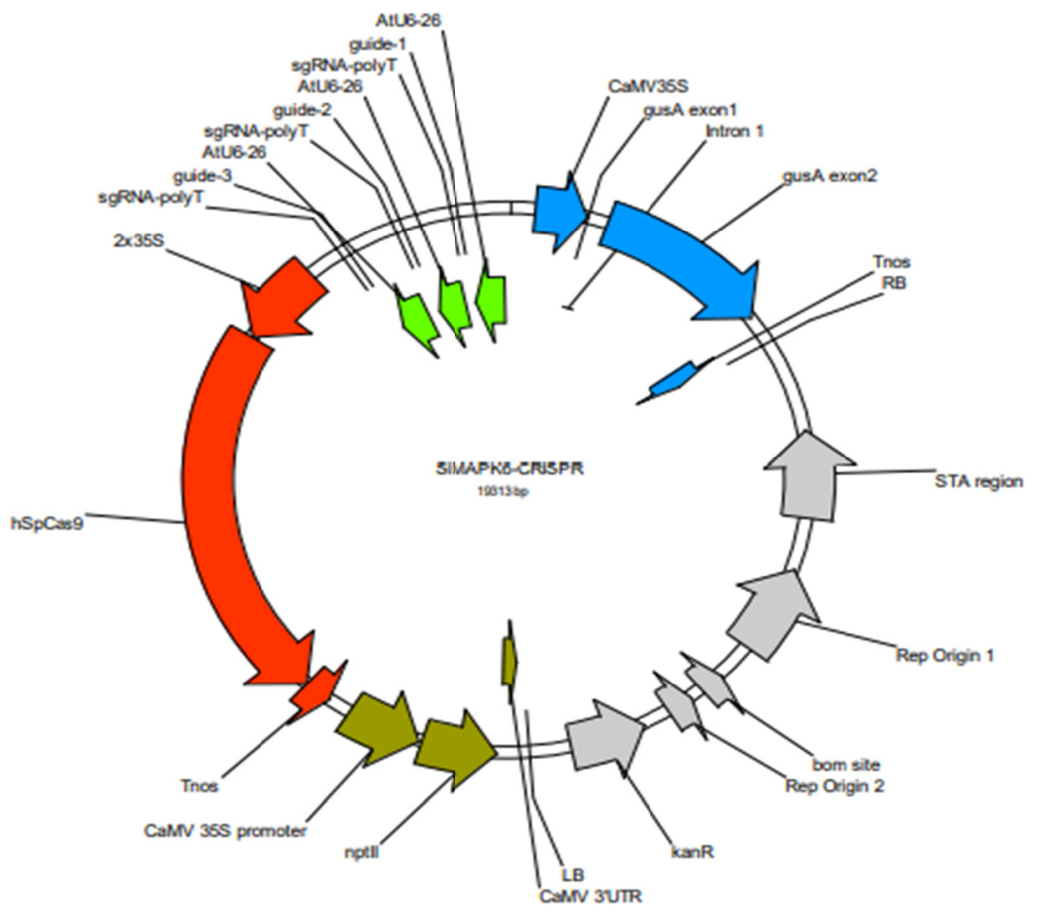

\section{Copyrights}

Copyright for this article is retained by the author(s), with first publication rights granted to the journal.

This is an open-access article distributed under the terms and conditions of the Creative Commons Attribution license (http://creativecommons.org/licenses/by/4.0/). 